\title{
MODELLI ITALIANI E FRANCESI A CONFRONTO NELLA GRAMMATICA CLASSICA DELLA LINGUA FRANCESE DI BOUDET DE MONTESQUIEU (1832)
}

\author{
ANNE SCHOYSMAN (*)
}

SunTO. - Pur riferendosi alla tradizione di Dumarsais e Condillac, Boudet de Montesquieu è tra i primi grammatici a dichiarare esplicitamente «che per imparar bene una qualunque siasi lingua, non vi abbia mezzo migliore che accennare allo studente in che la sua lingua si assomigli a quella che egli impara». Si mostra come questo metodo comparativo, nuovo nella storia della didattica del francese in Italia, rivela profonde divergenze per quanto riguarda la concezione della norma per ciascuna delle due lingue.

$* * *$

ABSTRACT. - Although he refers to the tradition of Dumarsais and Condillac, Boudet de Montesquieu is among the first grammarians to declare explicitly that the best way to help a student learn a new language well is to explain the similarities and differences between his own language and the language he is learning. This contribution shows how this comparative method, new in the history of French didactics in Italy, reveals profound differences regarding the conception of the norm for each of the two languages.

Nel 1832 viene pubblicata a Prato un'opera intitolata Grammatica classica della lingua francese, con esempj tratti da' migliori autori francesi, scritta ad uso degli Italiani, composta in italiano da Boudet de Montesquieu, avvocato. ${ }^{1}$ Di questo giurista dedicatosi alla grammatica

(*) Università di Siena, Italia. E-mail: anne.schoysman@unisi.it

1 Grammatica classica della lingua francese, con esempj tratti da' migliori autori francesi, scritta ad uso degli Italiani dal Professor Avvocato Boudet de Montesquieu, Dottor dell'Università di Francia, Membro della società di Giurisprudenza, Prato, Giachetti, 1832. L'opera è stata ristampata a Firenze, presso Ricordi e Comp., nel 1834, 
sappiamo ben poco, ${ }^{2}$ se non che quando intraprende il suo lavoro vive da «sol da quattro anni» a Firenze:

L'avere io voluto imprendere a paragonar la lingua mia coll'italiana favella, non sarà egli fiera temerità a me siccome colui il quale sol da quattro anni me ne sto nell'egregia città di Firenze?

Ci interesserà qui proprio l'impostazione contrastiva di questa Grammatica redatta da un Francese con l'intenzione di «paragonar la [s]ua lingua coll'italiana favella», perché appare rivelatrice della coscienza teorica e metodologica dell'eteroglossia dell'autore. Nel primo terzo dell'Ottocento non sono pochi gli editori o traduttori di grammatiche francesi in Italia, ma questi, come ha mostrato Nadia Minerva ${ }^{4}$ si limitano generalmente a riproporre i testi di François de Wailly, di Charles François Lhomond, di Sylvestre de Sacy o altri, senza modifiche. Per contrasto, spicca un caso relativamente eccezionale come quello di Giovan Battista Melchiori, adattatore nel 1814 della Grammaire philosophique et littéraire dell'abate Lévizac, con numerose aggiunte, «essendo ben altro il dettar precetto di lingua nella propria favella $[\ldots]$ ed altro l'insegnare a' medesimi una lingua straniera».5

poi nel 1839 con il titolo: Grammatica classica della lingua francese, con 2000 esempj tratti dai migliori autori francesi, colla giunta d'un indice generale [...]. [d'ora in poi: BOUDET 1832, BOUDET 1839].

2 Il suo nome compare tra i corrispondenti di Giovan Pietro Vieusseux, per un unico scambio di lettere, nel 1837: cf. Letizia PAGLIAI, Repertorio dei corrispondenti di Giovan Pietro Vieusseux, dai carteggi in archivi e biblioteche di Firenze (1795-1863), Firenze, Leo S. Olschki, 2011, p. 55.

3 BOUDET 1832, p. 7; corsivo dell'autore. Si può tuttavia sospettare che Boudet de Montesquieu sia ricorso ad un revisore perché, come conferma Giuseppe Patota, che ringrazio, nella prefazione dell'autore non si rintracciano forme che non siano riconducibili all'italiano scritto di primo Ottocento.

4 MinerVA, Nadia, «Les manuels italiens des années 1800-1860 et l'enseignement de la langue française», in Herbert CHRIST, Gerda HASSLER (éds.), Regards sur l'bistoire de l'enseignement des langues étrangères. Actes du colloque de la SIHFLES au Romanistentag de Potsdam du 27 au 30 septembre 1993, Tübingen, Gunter Narr Verlag, 1995, pp. 82-92 [d'ora in poi: MiNERVA 1995], p. 86. Per la storia delle grammatiche francesi in Italia, vedere MinERVA, Nadia; PELLANDRA, Carla, Insegnare il francese in Italia. Repertorio analitico di manuali pubblicati dal 1625 al 1860, Bologna, CLUEB, 1997 (ed. aumentata; $1^{a}$ ed. 1991).

5 Giovanni Battista Melchiori, Grammatica filosofico-letteraria della lingua 
Ritroviamo questa stessa preoccupazione nella grammatica di Boudet de Montesquieu, che presenta inoltre la particolarità di essere non un adattamento, bensì un'opera originale:

E sì come mi do ragionevolmente a credere che per imparar bene una qualunque siasi lingua, non vi abbia mezzo migliore che accennare allo studente in che la sua lingua si assomigli a quella che egli impara, $\mathrm{e}$ in che sì l'una che l'altra sieno differenti, così ho procurato, secondo mia debole possa, di rilevar con esattezza ove la favella francese colla italiana s'accordi, e dove l'una discordi dall'altra. ${ }^{6}$

Oltre all'impianto contrastivo della sua grammatica, Boudet de Montesquieu dichiara anche i suoi modelli teorici, insistendo sulla loro novità in Italia:

[...] diversi maestri han fatto dono all'Italia di varii trattati sulla lingua francese, onde agevolarne vie [sic] più lo studio. A me però sembra che fra la moltitudine di que' trattati non ve n'abbia pur uno in cui le regole ed i precetti vengano raccolti ed insieme distribuiti secondo il nuovo sistema d'analisi e d'ideologia in uso a' dì nostri, e segnatamente presso del' Francesi. $^{7}$

Il riferimento ai grammatici «idéologues» e «philosophes» è esplicito: i modelli dichiarati di Boudet de Montesquieu «per quella parte che riguarda la filosofia della lingua» sono Du Marsais ${ }^{8}$ e Condillac, " «a'

francese tratta dalla recente del sig. abate di Lévizac, Parigino, e da' più celebri scrittori francesi, con aggiunte utilissime per gl'Italiani, Brescia, Bendiscioli, 1814, p. VIII, cit. in MineRVA 1995, p. 86.

6 BOUDET 1832, p. 7.

7 BOUdET 1832, p. 3.

8 Boudet de Montesquieu ha potuto consultare i testi di César Chesneau Du Marsais riuniti in edizione postuma sotto il titolo Logique et principes de grammaire (Paris, Briasson, 1769; in 2 t., Paris, Barrois, 1792); per una edizione recente, v. César Chesneau Du MARSAIS, Les véritables principes de la grammaire et autres textes (17291756), éd. Françoise DouaY-Soublin, Paris, Fayard, 1987.

9 Nel Discours Préliminiare al suo Cours d'étude pour l'instruction du prince de Parme, il cui primo tomo è una Grammatica redatta alla fine degli anni 1650, Condillac presenta un metodo pedagogico mirato a «apprendre à penser» (BIONDI, Carminella, «La lunga premessa di Condillac: per un corretto uso del Cours d'études», in PellandRA, Carla; VINEIS, Edoardo, Grammatiche, grammatici, grammatisti. Per una 
quali si dà il vanto del metodo analitico». ${ }^{10} \mathrm{Il}$ grammatico giustifica così la scelta di trattare insieme, nella prima parte della sua grammatica, «l'etimologia» (ossia «il vero significato e proprio delle voci che la nostra orazione compongono») e la «sintassi»:

io mi credo sia più conforme sì allo spirito d'analisi, sì all'ideologia, lo scriversi in uno coll'etimologia per entro il titolo d'ogni parte dell'orazione, tutte le regole della sintassi, le quali ad esso titolo s'appartengono, anzi che farne un trattato a parte; [...] un cotal metodo non è mica nuovo; esso vien praticato da' più celebri ideologi francesi. ${ }^{11}$

Da questa filosofia deriva direttamente la necessità di fondarsi non su esempi forgiati espressamente in frasi-sentenze per illustrare le varie regole grammaticali, come era prassi corrente, ${ }^{12}$ ma sull' «l'autorità degli autori e la ragione», offrendo e discutendo esempi letterari: «Sicuri sono i testi citati in questa opera, perché tratti da corrette edizioni, ed holli io tratti dagli autori medesimi, non presili da verun grammatico». ${ }^{13}$ Il titolo

storia dell'insegnamento delle lingue in Italia dal Cinquecento al Settecento, Pisa, Goliardica, 1989, pp. 161-177, p. 166), così come Boudet de Montesquieu intende «dare in luce una Grammatica ragionata della lingua francese, la quale discostandosi dal rancido metodo fin qui praticato, e trattando il linguaggio filosoficamente, insegni allo studente ad imparare non che a riflettere» (BOUDET 1832, pp. 3-4; sottolinea l'autore). «C'est à l'usage des mots», dice Condillac, «que vous devez le pouvoir de considérer vos idées chacune en elle-même, et de les comparer les unes avec les autres pour en découvrir les rapports. [...] On se tromperait par conséquent si l'on croyait que les langues ne nous sont utiles que pour nous communiquer mutuellement nos pensées. C'est donc comme méthodes analytiques que nous les devons considérer» (Étienne Bonnot de Condillac, cit. in RICKEN, Ulrich, «Teoria linguistica e sovversione ideologica: La Grammaire di Condillac e la censura del suo Cours d'études da parte delle autorità ecclesiastiche di Parma», in Formigari, Lia; LO PIPARO, Franco (éds.), Prospettive di storia della linguistica, Roma, Editori Riuniti, 1988, pp. 241-255, p. 247).

10 BOUDET 1832, p. 5.

11 Ibid.

12 Nel sul studio sui manuali italiani degli anni 1800-1860 e l'insegnamento della lingua francese, Nadia Minerva ha evidenziato come si contrappongano, in quegli anni, essenzialmente due tipi di grammatiche: le opere destinate alla consultazione, che aspirano ad una descrizione ragionata ed esaustiva della lingua dei grandi autori, e le opere pedagogiche destinate all'apprendimento sistematico, con esercizi progressivi (MineRva 1995, pp. 84-85). La Grammatica Classica Di Boudet de Montesquieu appartiene ovviamente al primo gruppo.

13 BOUdET 1832, p. 6. 
dell'edizione del 1832 menziona «con esempj tratti da' migliori autori francesi», e quello della ristampa del 1839 sottolineerà: «con 2000 esempj tratti dai migliori autori francesi». ${ }^{14}$

Sono messi a contribuzione Fénelon - il grande e costante modello della prosa classica -, La Rochefoucauld, La Bruyère, Massillon, Voltaire, Boileau, Bossuet, La Fontaine, Montesquieu, Molière, l'Académie Française, Pascal, Racine, D'Aguesseau, Vauvenargues, Nicole, Corneille, Regnard. ${ }^{15}$ Si tratta, senza sorpresa, del canone dei classici del Seicento, momento di cristallizzazione, almeno teorica, della norma linguistica in Francia. È ovviamente in questo senso che Boudet de Montesquieu giustifica il titolo di Grammatica classica.

Tuttavia, nel principio della «grammatica ragionata della lingua francese ${ }^{16}$ che è il suo, Boudet de Montesquieu si avvale degli esempi d'autori classici senza mai perdere di vista lo scopo di individuare, al di là delle «diversità di opinioni», «i precetti ed i principj» che debbano avere valore di norma anche indipendentemente dalla varia pratica degli scrittori. Nella sua Prefazione leggiamo:

In quella parte poi che riguarda l'uso e la pratica della lingua, ch'è quanto dire, $i$ precetti ed $i$ principj, io mi sono mai sempre appigliato all'opinion de' migliori grammatici francesi, qualora vien corroborata dall' autorità sì de' Classici, che dell'Accademia francese. Trovatasi diversità di opinioni tra' grammatici, io holle insieme comparate con qualche attenzione, e sonomi appligliato al parere, che m'è paruto più solido e più conforme $a^{\prime}$ principj. E se tal fiata mi son presa la libertà di contradire alle opinioni loro, ho creduto di poterlo fare, quando ho avuto in favor mio l'autorità degli autori e la ragione. ${ }^{17}$

Questa «libertà di contradire» l'uso per trarne principii normativi non era scontata e situa decisamente Boudet de Montesquieu dalla parte dei grammatici «idéologues», se si pensa che per esempio GiraultDuvivier, pur basando anche lui la sua celebre Grammaire des grammaires su citazioni d'autore, rifiutava di prendere posizione sui fatti gram-

14 Cf. Carla Pellandra, «Lezioni di lingua, lezioni di morale: gli esempi d'autore della Grammatica classica di Boudet de Montesquieu (1839)», in Nadia Minerva; Carla Pellandra (éds.), Aspetti di etica applicata. La scrittura aforistica, Bologna CLUEB, 2000, pp. 287-300 [d'ora in poi: PELLANDRA 2000].

15 PELlandRa 2000, pp. 292-293.

16 BOUDET 1832, p. 3.

17 BOUDET 1832, pp. 5-6; sottolineo io. 
maticali che l'uso lasciava indecisi. ${ }^{18}$ Boudet de Montesquieu ha del resto voluto ribadire questo punto nella prefazione alla riedizione della sua Grammatica classica nel 1839, dove appare anche più evidente quanto l'autorità degli scrittori, pur necessaria a corroborare l'«opinion de' migliori grammatici francesi» e dell'Accademia, sia comunque subordinata «alla sana grammatica», ossia ad una «regola»:

Non negherò essermi fatto lecito il riprendere ne' Classici nostri, quel che alla sana grammatica m'è paruto non confarsi, acciocché lo studioso abbattendosi in un qualche irregolare esempio d'autore Classico, non s'avvisi per avventura essere la regola nostra contraria al Classico, e non il testo autorevole alla regola attraversare. Nè per questo si dia alcuno a credere ch'io m'abbia voluto far l'aristarco della mia lingua, per spirito di pedanteria, o per inzigamento di critica. Mi piacque sempre il rispetto verso i celebrati scrittori, di che fa solenne testimonianza il titolo dell'opera mia; ma però non bisogna nè ficcarsi in testa ch'essi non mai potessero errare, nè voler pertinacemente sostenere ogni cosa detta da loro. [...] Del resto, ove m'avvenga di arrischiare la mia critica in su de' Classici, valga a difendermi l'ingenuina osservazione del Muratori: Potere anche gl'ingegni minori scoprire talvolta quelle macchie che i maggiori non hanno saputo scoprir o schivare nelle proprie cose; e non essere necessaria quella stessa forza di mente per la teorica delle arti, che si richiede poscia alla lor pratica. E qui mi piace arrogere per mio contento che tanto minor meraviglia deesi destare da questo criticar mio, in quanto che esso restrignesi a solamente opporre, quando un Classico all'un altro, quando il Classico a sè medesimo. ${ }^{19}$

18 Nella sua prefazione, Girault-Duvivier aveva dichiarato: «j'ai cherché à réunir en un seul corps d'ouvrage tout ce qui a été dit par les meilleurs Grammairiens et par l'Académie, sur les questions les plus délicates de la langue française. Je me suis rarement permis d'émettre mon avis; j'ai dû me contenter de rapporter, ou textuellement, ou par extrait, celui des gands maîtres [...]. J'ai indiqué avec une scrupuleuse exactitude les sources où j'ai puisé ; j'ai mis en parallèle les opinions des différents auteurs, mais j'ai laissé aux lecteurs le droit de se ranger à tel ou tel avis, lorsque la question restoit indécise, ou que la solution n'en étoit ni indiquée par l'analogie, ni donnée par l'usage le plus généralement adopté [...]. J'ai laissé aux idéologues et aux métaphysiciens le soin de démontrer ce qu'ils trouvent de vicieux ou de faux dans les anciens termes, et la gloire d'en proposer de nouveaux» (Charles-Pierre GIRAULT-DuviviER, Grammaire des grammaires ou analyse raisonnée des meilleurs traités sur la langue française, Paris, Janet et Cotelle, 1827 [1 $1^{\mathrm{a}}$ ed. Paris, Porthmann, 1811], Préface, p. I-II, VIIVIII; d'ora in poi: GIRAULT-DUVIVIER 1827).

19 BOUDET 1839, pp. 6-7; sottolineo io. 
Nel presentare questo suo metodo, Boudet de Montesquieu non fa cenno dell'impostazione comparata della sua grammatica, pur dichiaratamente incentrata sul «paragonar la lingua [sua] coll'italiana favella». ${ }^{20} \mathrm{Ma}$ non stupirà che egli scelga, anche per l'italiano, esempi letterari tratti dai maggiori autori invece di frasi anonime forgiate dai grammatici, né stupirà che il periodo di riferimento sia il Trecento-Quattrocento di Dante, di Giovanni Villani, di Boccaccio, Petrarca o Passavanti, cioè il periodo del cristallizzarsi del modello linguistico toscano così come il Seicento francese si è imposto a modello per la stabilizzazione della lingua nazionale. Il doppio criterio adottato da Boudet de Montesquieu, quello di una «grammatica classica» con le caratteristiche che abbiamo riassunte, insieme a quello di una grammatica comparativa, crea e spiega quello strano effetto di prospettiva per cui il francese illustrato con i classici del Seicento viene descritto attraverso comparazioni con l'italiano dei grandi Toscani del Trecento. Basteranno pochi esempi (in grassetto gli autori citati):

I nomi proprii sendo di per se abbastanza determinati, non han mestieri d'articolo. Pâris n'aurait pas enlevé Hélène (Fénelon). Sophocle, Euripide, Térence, Homère et Virgile nous sont encore en vénération (Racine). Virgile, Varius, Pollion, Horace, Tibulle étaient amis (Voltaire) ${ }^{21}$ Il che usarsi nella lingua italiana apertamente si vede dal seguente esempio di Boccaccio (introd. del suo decam.): Li quali non che altri, ma Galieno, Ippocrate, o Esculapio avrieno giudicati sanissimi. ${ }^{22}$

Noteremo che tal fiata nella lingua toscana trovasi il positivo replicato, il quale conviene ridursi al superlativo. Addurronne un esempio di Boccaccio [novella V, 10]: Elle si vorrebbon vive vive metter nel fuoco. Nè mancano presso di noi esempli di così replicato positivo. E ne fa fede il seguente testo di Fénelon: Il était une fois une vieille si vieille, si vieille qu' elle n'avait plus ni dents ni cheveux (Fable 7). ${ }^{23}$

Vous et moi nous sommes contents de notre sort (Académie française) $;{ }^{24}$ Je pense que vous et moi nous avons été les seuls (Voltaire); Il et vrai qu'elle et moi sowvent nous nous sommes parlé des yeux (Molière); Nous et lui là-dessus nous sommes tous égaux (La Fontaine); Patrocle et moi, seigneur, nous irons l'assiéger (Racine); Votre père et moi nous

20 BOUdET 1832, p. 7.

21 Esempio presente in GIRAULT-DUVIVIER 1827, p. 649.

22 BOUDET 1832, p. 31.

23 Boudet 1832, pp. 193-194.

24 Esempio presente in Girault-Duvivier 1827, p. 340. 
avons été longtemps ennemis l'un de l'autre (Fénelon). Notasi che il tralasciare nous, vous in simil caso, comecchè ve n'abbia esempli non pochi ne' nostri classici, non è mica da usarsi; ed a sì fatta omissione si oppongono i nostri grammatici, e a me pare che abbiano ragione.

[In nota] Pare esser proprietà della lingua toscana il non esprimere noi, voi nel caso di cui si tratta. E fede ne fanno Dante, Petrarca, Boccaccio: Lo Duca ed io per quel cammino ascoso entrammo (Infern. 34); Poichè voi ed io più volte abbiam provato (Petrarca, Sonetto 67 [99]); Io gli farò giuoco, che nè tu nè egli sarete mai lieti (Boccaccio, VIII, 8) [ecc.] $]^{25}$

È sintomatico che di questa sfasatura cronologica tra esempi italiani ed esempi francesi Boudet de Montesquieu non faccia cenno: questo silenzio rivela l'ovvietà del canone linguistico adottato. Il metodo che porta Boudet de Montesquieu a mettere a confronto il francese e l'italiano dei «grandi autori» delle rispettive lingue giustifica perfettamente l'accostamento dei «classici» del Seicento francese con i Toscani del Trecento. Del resto, l'italiano letterario di cui fa uso Boudet de Montesquieu nella redazione della sua grammatica non fa che riflettere lo stesso canone.

Ma per quanto riguarda il trattamento delle «diversità di opinioni tra' grammatici», di «qualche irregolare esempio d'autore» sul quale Boudet de Montesquieu rivendica di «arrischiare la [sua] critica in su de' Classici», con quali criteri avviene la scelta degli esempi d'autore italiani messi a confronto al fine di illustrare la specificità della «regola» francese? Proprio questo punto ci permette di illustrare la maniera in cui Boudet de Montesquieu descrive e concepisce le peculiarità di ciascuna delle due lingue.

Si partirà dalla costatazione, ovvia ma non senza conseguenza sulla descrizione grammaticale, che Boudet de Montesquieu espone le regole del francese, alle quali solo in un secondo tempo contrappone l'uso italiano. La stessa disposizione tipografica dell'opera ne testimonia, essendo le considerazioni sull'italiano molto frequentemente inserite in nota a pié di pagina. In una grammatica comparata questa impostazione non è indifferente, poiché si evidenzia in questo modo come l'italiano si differenzi dal francese, il che corrisponde di fatto al punto di vista del Francese Boudet de Montesquieu, e non come il francese si differenzi dall'italiano, che dovrebbe essere invece la prospettiva del pubblico della Grammatica classica della lingua francese scritta ad uso

25 BOUDET 1832, pp. 70-71. 
degli Italiani. Si veda per esempio la conclusione del capitolo dedicato al Nome: dopo aver trattato il genere, il numero, e «l'estensione di significato» (senso determinato, indeterminato, partitivo) che sarà dato dagli articoli, Boudet inserisce a pié di pagina una lunga nota dedicata ai suffissi italiani e alla loro traduzione in francese:

Per non ometter nulla di quanto s'appartiene al nome, rimane il dir qualcosa de' nomi accrescitivi, peggiorativi e diminutivi proprj della toscana favella. Di tai nomi che son per quella lingua fonti perenni sì di dovizie, sì d'energia, viene affatto priva la lingua francese, non avendone più di dieci, o in quel torno. Per la qual cosa fa di mestieri ricorrere ad uno o più addiettivi, onde esprimere in francese il vero significato di così fatti nomi. [...]. La terminazione one esprime volume, grandezza; egli converrà aggiungere l'addiettivo grand al nome. [...] De' peggiorativi le terminazioni sono accio, azzo, astro, le quali indicano l'oggetto come laido, cattivo. Onde si aggiungerà al nome l'addiettivo vilain o l'addiettivo mechant [...]. Quanto a' diminutivi ricchissima n'è la favella toscana, e varie sono le terminazioni di così fatti nomi. ${ }^{26}$

Ma questa impostazione ha anche una incidenza sulla descrizione stessa della lingua italiana. Dato che il «buon metodo grammaticale» francese propugnato da Boudet de Montesquieu mira a individuare la regola, la forma corretta da adottare mentre altre varianti non conformi a «'autorità degli autori e la ragione» sono da evitare, il confronto sia tra esempi francesi, sia tra francese e italiano, risulta assai complesso, soprattutto quando la comparazione porta su fatti sintattici: nel suo capitolo sulla «Replicazione dei pronomi», l'autore avverte che «non è senza difficoltà la materia che imprendiamo a trattare, sendo cotanta la differenza di sintassi che in su di ciò trovasi in ambo le lingue». ${ }^{27} \mathrm{E}$ tutte le volte che il grammatico contrappone al francese l'italiano, finisce immancabilmente col notare una maggior libertà d'uso nell'italiano. Per esempio, dopo aver speso alcune pagine sulle regole della sintassi dei pronomi francesi se e soi, sulla loro posizione rispetto al verbo, sulla natura del soggetto, persona o cosa, a cui si riferiscono, e sull'opinione dei grammatici in merito, ${ }^{28}$ Boudet de Montesquieu conclude che «più franca sì e più spe-

\footnotetext{
26 BOUDET 1832, p. 19.

27 BOUdET 1832, p. 64.

28 BOUdET 1832, pp. 57-61.
} 
dita è la sintassi del pronome se italiano, sì come quello che senza patire alcuna cosa d'eccezione, serve ad ambo i generi nell'uno e nell'altro numero, particolare o generale che sia il soggetto della frase». ${ }^{29} \mathrm{O}$ ancora, il francese deve replicare il pronome davanti a più verbi coordinati,

ed error manifesto di lingua sarebbe il tralasciare il pronome, come a dire: il m'aime et honore, in vece di il m'aime et m'bonore. Onde si dirà con essi i nostri classici: Ils nous abordent, nous prennent, et nous emmènent prisonniers en Égypte (Fénelon); Octave se conduisit avec Cicéron en homme habile: il le flatta, le loua, le consulta (Montesquieu); Le feu brille dans leurs yeux, ils se raccourcissent, ils s'allongent, ils se baissent, ils se relèvent, ils s'élancent (Fénelon),

ma aggiunge Boudet de Montesquieu in nota:

Pare esser lecito dietro agli scrittori Toscani il non replicar il pronome in simil caso. E il Caro, per tacer di tanti altri, ha detto in fine di una sua a Anton Simone Notturno: Ed a voi per sempre m'offero e raccomando. ${ }^{30}$

Ne esce sistematicamente un ritratto dell'italiano come una lingua molto meno assoggettata del francese a regole grammaticali. La cosa si fa ancora più palese quando Boudet de Montesquieu, intento a «ridurre la lingua a vero modello grammaticale» e a individuare le «vere regole» della lingua francese dettate dalle opinioni dei grammatici dalla «ragione», si impegna a sanare le diversità di uso degli scrittori francesi stessi, confrontando, correggendo o condannando anche grandi autori classici. Per esempio, sempre nel capitolo che tratta della sintassi dei pronomi, Boudet enuncia come regola inderogabile l'obbligo di:

sempre premettere al verbo il pronome plurale nous, vous, secondo occorre, ossia che i pronomi oggetti al verbo succedano, ossia che ad esso premettansi. Onde si dirà: vous nous appelez lui et moi.

La fortune nous a persécutés lui et moi (Fénelon)

Ma gloire loin d'ici vous et moi nous appelle (Racine).

Notisi che l'omettere nous, vous in simil caso, quantunque vi abbia esempli d'ottimi autori i quali usarono tacere essi pronomi, dee reputarsi error manifesto di lingua. E tale è l'opinione de' più celebri grammatici francese. Errò adunque Fénelon, quando scrisse (Télémaque, Liv. 7):

29 BOUDET 1832, p. 61.

30 BOUdET 1832, p. 72. 
Pénélope ne voyant revenir ni lui ni moi. Dovea premettere il pronome nous innanzi a voyant e dire: ne nous voyant revenir ni lui ni moi. ${ }^{31}$

In questa spiegazione colpisce l'imputazione di errore a Fénelon, pure considerato come uno «de' più perfetti scrittori della lingua francese»; ${ }^{32}$ ma immediatamente dopo, Boudet de Montesquieu introduce il paragone con l'italiano, non soggetto a questa regola, secondo l'autorità del Boccaccio:

Pare che nella lingua toscana i pronomi noi, voi possansi tacere in questo caso; e fanne fede l'autor del Decameron (II, 3): E puoi veder me e la famiglia dormir su per le panche. E nella stessa giornata, n[ov]. 6: il quale e lui e me prese in corso.33

\section{Allo stesso modo, Boudet de Montesquieu dichiara che:}

il tralasciare le, la, les (il che diremo per passaggio, è lecito appo gli scrittori Toscani), benché non manchino esempli di buoni autori ed approvati, è error manifesto di lingua, anzi che no. Ed essa opinione è a dirla non già il sentimento di me solo, ma sì del bene di tutti quanti i grammatici che antichi che moderni. Fallarono adunque Bossuet e Racine quando scrissero:

[Bossuet]Il semble que Vado ait eu un bon dessein, et que la gloire de la pauvreté ait séduit lui et ses partisans.

[Racine] Cela pourra réjouir elle et mon fils (Lettre à Boileau).

Dietro alla nostra regola ad ognuno è palese che Bossuet dovea premettere il pronome le innanzi a ait séduit; Racine il pronome la avanti a réjouir. ${ }^{34}$

Ancora una volta, un Bossuet o un Racine possono commettere errori se sono dichiarati tali dai grammatici, ${ }^{35}$ mentre quel che è «error

31 BOUDET 1832, pp. 74-74.

32 BOUdET 1832, p. 74, nota 1.

33 BOUDET 1832, p. 74.

34 BOUDET 1832, pp. 75-76.

35 Per esempio Jean-François Féraud, autore di un Dictionnaire critique de la langue française (Marseille, 1787-1788). Féraud è citato nella Grammaire des grammaires di Girault-Duvivier, dalla quale Boudet de Montesquieu riprende numerosi esempi, tra i quali il commento di Féraud affermando che Bossuet avrebbe dovuto scrivere «l'ait séduit, lui et ses partisans», e Fénelon «ne nous voyant revenir ni lui ni moi» 
manifesto di lingua» in francese «è lecito appo gli scrittori Toscani» secondo la testimonianza del Boccaccio, come annota Boudet de Montesquieu:

E in pruova mi si presentano i seguenti passi di Boccaccio:

E con grandissima festa lei e'l suo genero ricevette (II, 3).

E nella nov. 8 della stessa giornata:

A perpetuo esilio lui e $i$ suoi discendenti dannarono. ${ }^{36}$

Nel valore accordato alle citazioni d'autore, Boudet de Montesquieu usa, di fatto, due pesi e due misure: i pur «ottimi autori» francesi sono suscettibili di commettere «error manifesto di lingua»; per contrasto l'uso dell'italiano, testimoniato dall'autorità degli autori dalla sola autorità degli autori, non sottoposti al confronto tra di loro o con una norma derivata dall'«opinion de' migliori grammatici» - non risulta sottoposto alle rigide regole sintattiche del francese. In conclusione, appare evidente come sia proprio la difficoltà di conciliare il «buon metodo grammaticale» con una impostazione comparativa a rivelare profonde divergenze nella concezione della norma in ciascuna delle due lingue. Per il francese, la Grammatica classica, che si vuole «grammatica ragionata» e rivendica la possibilità, per il suo autore, di «riprendere ne' Classici nostri, quel che alla sana grammatica m'è paruto non confarsi», ${ }^{37}$ rispecchia perfettamente quella stagione normativa che ha determinato una concezione largamente codificata della lingua, in particolare per quanto riguarda i fatti sintattici, a riprova del fatto risaputo che la descrizione grammaticale è un fatto eminentemente culturale. ${ }^{38}$ Tuttavia nel confrontare i parere dei grammatici e i molti esem-

(Girault-Duvivier, Grammaire des grammaires, éd. 1827, cit., p. 355). Ma Boudet de Montesquieu ha potuto leggere il commento di Féraud all'esempio di Bossuet anche nell'opera di Le François de Hautevesne, anche se quest'ultimo è piuttosto polemico: «suivant Féraud, il faudrait que le pronom de la personne précède le verbe; cet auteur prétend que Bossuet devrait dire: Il semble que la gloire de la pauvreté l'ait séduit lui et ses partisans. Cette construction ne vaut rien». (Ch.-Ant. LE FRANÇOIS DE HAUTEVESNE, Grammaire universelle classique et polémique, Paris, Bossange Père, 1824, p. 164).

36 BOUDET 1832, p. 75, nota 1.

37 BOUdET 1839, p. 6.

38 Data la vasta bibliografia su questo argomento, ci si limiterà a citare qui un contributo di sintesi: HASSLER, Gerda, «La description du génie de la langue dans les grammaires françaises et les grammaires d'autres langues», in Bernard COLOMBAT, 
pi di classici francesi, l'autorità e gli «errori manifesti» di un Racine o di un Fénelon, con il toscano illustrato dai grandi prosatori del Trecento, Boudet de Montesquieu finisce implicitamente e paradossalmente col rivelare della grammatica italiana una concezione meno rigidamente normata, ma anche molto più uniforme, più statica, meno dibattuta dai grammatici di quella della grammatica francese.

Jean-Marie FOURNIER, Valérie RABY (éds.), Vers une histoire générale de la grammaire française. Matériaux et perspectives, Paris, Honoré Champion, 2012, pp. 193-209. 